\title{
THE PRESTO PROJECT RELAY: OPEN, ASYNCHRONOUS LEARNING IN VIRTUAL PEER GROUPS
}

Pieter W. G. Bots, C. Els van Daalen, Sofia M. Dopper, Delft University of Technology, The Netherlands

\begin{abstract}
Academic education entails that students engage in open-ended assignments. Global education entails that students interact in projects with peers world-wide. The logistic challenge of offering academic project work to a large, heterogeneous student body, while keeping instructor workload manageable, is daunting. Project relays on the PRESTO software platform meet this challenge. In a project relay, students work in a virtual peer group on assignments that are organized in successive steps, where each step is peer reviewed. Unlike a regular peer review, the reviewing students revise the work they have reviewed. They then add the next step to it, and pass the improved-and-extended work on to another student. The PRESTO software fully automates the relay workflow, facilitates defining, monitoring, and grading projects, and has been adapted for use in LTI-compliant MOOCs. Since 2013, over 20 project relays have run in several courses at Delft University of Technology. Evaluations show that a project relay realizes the intended learning outcomes, but can at times be stressful for students. This prompts ideas for further pedagogical and technical improvements.
\end{abstract}

\section{Introduction}

Courses that aim at attaining Bloom's application level of learning typically require repeated practice from students. For some subjects, exercises can be devised that have a single correct result, but to achieve deep learning, students must work on open-ended assignments (Ke \& Xie, 2013). Providing feedback on, and assessing, open-ended assignments (e.g., writing an essay or reporting on a problem analysis) requires interpretation and judgement, which makes courses very teacher-intensive. Reducing teacher load by conducting the work in project groups introduces other problems, such as free rider behaviour (Brooks \& Ammons, 2003). Faced with the challenge to provide students with sufficient individual practice and feedback, while at the same time keeping the teaching burden manageable, we have developed the project relay method and its supporting software (PRESTO).

The project relay was originally developed for teaching first-year students the basics of constructing quantitative models. Since then, it has been adapted to support any type of project work that can be partitioned into consecutive steps, e.g., writing an essay (literature review $\rightarrow$ thesis statement $\rightarrow$ introduction $\rightarrow$ body of information $\rightarrow$ conclusion) or conducting a life cycle analysis (goal and scope $\rightarrow$ inventory analysis $\rightarrow$ impact assessment $\rightarrow$ 
interpretation). The higher level skills required for these activities cannot be learned by only studying a textbook, but require learning by doing.

The objective of this paper is to explain how the relay system can be used by students and instructors. In order to do this, we first introduce the relay method and present the key features of the PRESTO software. We then briefly report some of our experiences using project relays in several courses, argue that it affords open, asynchronous learning in virtual groups across educational institutions, and outline our plans for future development.

\section{The project relay method}

The assignments students carry out in a relay are divided up into a number of consecutive steps. In all but the first step, students receive - similar to the relay baton in athletics completed preceding steps from an anonymous predecessor. As each step will concern a different case or topic, students cannot continue their own work on the previous step. Knowing that they have to perform their primary task on this new case gives students a natural incentive to immerse themselves in their predecessor's work. Having to review and if necessary - improve their predecessor's work before extending it with their "own" step makes that students gain a deeper understanding of their predecessor's work, allowing them to provide more substantive feedback to their predecessor. Moreover, having to "take ownership" of their predecessor's work makes that students receive additional practice in conducting the previous steps.

Figure 1 shows an example of a relay process in which students are asked to develop a quantitative model, given a written description of a system. The assignment is divided into 6 steps that follow on from each other, starting with a research question and ending in conclusions that can be drawn from running the computational model with different input values. In Step 1, students start from scratch, and submit work that is strictly their own. In each following step, students have to perform four tasks:

1. study the work which has been submitted by the predecessor,

2. provide constructive feedback and assess the work of the predecessor on a 5-point scale,

3. improve the work,

4. extend the work by adding their "own" step (primary task).

The result of the final step is then reviewed and assessed by two different successors. 


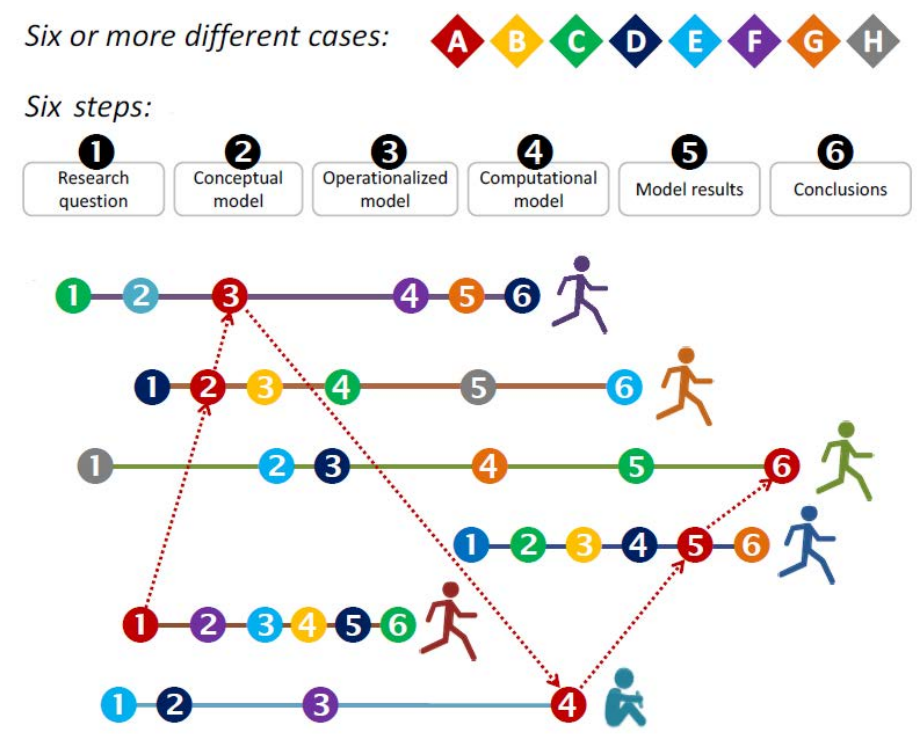

Figure 1. Schematic diagram of students going through the steps of a project relay

When starting with the first step, a student is assigned a randomly selected case. The result of the first step then goes to another student who has done Step 1 on another case. This student reviews and improves the work, and adds Step 2 to it. This work then goes to another student who just did Step 2 - also on another case. This student then reviews and improves the work, and adds Step 3. In Figure 1, the dotted arrows show how, in this process, an instance of case $\mathrm{A}$ is passed on from student to student. The horizontal lines reflect students performing asynchronously and at their own pace - consecutive steps on different cases. At the end of the relay, a student has worked on all steps for 6 different cases, and will have iterated several times through earlier steps because giving feedback and improving the work always relates to all previous steps. For a video explanation of the procedure, see https://youtu.be/SGmDstdElk.

It does not matter if a student drops out after a step, as this student will then not receive a case for the next step. Moreover, the relay system does not require a fixed schedule of who evaluates which work, since the work will be sent to the next person who is ready for an assessment. This allows students to do the steps of the relay at their own pace, as long as they finish the whole relay before the end date.

A downside of the asynchronous process is that students receive feedback on a step relatively late, because successors can still modify their review and assessment until they upload their own work. Their predecessor then gets to see the feedback (typically structured according to a rubric), and is invited to respond and appraise the feedback. In addition to the feedback, students can also view the work submitted by their successor to see how their own work has been modified and extended.

To ensure that students put in serious effort, we have introduced some specific incentives. The students know that after every step their work is assessed on a 5-point scale by peers, and - more importantly - that it will count towards their final grade following a differential scoring system. This means that students earn points if they score higher than (or maintain 
the high score of) their predecessor, and lose points if they score lower (or fail to improve on a low score). Although this motivates students to do their work well, it has as a perverse effect that it may lead students to underrate their predecessor's work. The PRESTO system therefore invites students to appeal if they feel that they have been unfairly assessed. When they appeal, a referee (typically an instructor or a qualified student) will judge the peer assessment, change it if needed, and impose penalty points on students rating too harshly or raising unfounded appeals.

Even with these incentives, it may happen that students receive work from a predecessor that is below par and would require a disproportionate amount of time to improve. For these situations, we allow students to reject such work. They then immediately receive the work of a new predecessor. Since the students must justify rejection with a thorough review, to which their predecessor can still appeal, this effectively prohibits rejection merely to get better work to build on.

\section{The PRESTO software}

The PRESTO software manages the workflow of a project relay, and facilitates defining, monitoring, and grading projects. It has been programmed in Python on the open source platforms Django and Semantic UI, and will be available under an open source license. The software discerns four user roles: student, instructor, developer and administrator. Students can enrol in courses, are led through relay steps and invited to respond to reviews for ongoing relays, and can view past relays in their personal history. Instructors can develop and share case sets (for use in different relays), create and operate relays for their courses, monitor progress, and grade students. Developers can design and share templates that define step sequences and specify what a student has to do within every step. Administrators can create courses, and assign instructor and developer roles.

\section{Student interface}

The student interface leads students through the tasks that have to be performed in each of the different steps: commit to "the rules of the game", read first assignment and case, upload own work, proceed to next step, download predecessor's work, review and assess predecessor's work, and upload own (new) work again. These tasks are "colour-coded" (e.g., green $=$ proceed, blue $=$ download, orange $=$ review), and are always carried out in the same sequence. Students first see information about the task for their current step, possibly followed by other tasks (responding to reviews of prior steps, and to referee decisions). 
The PRESTO Project Relay: Open, Asynchronous Learning in Virtual Peer Groups

PieterW. G. Bots et al.

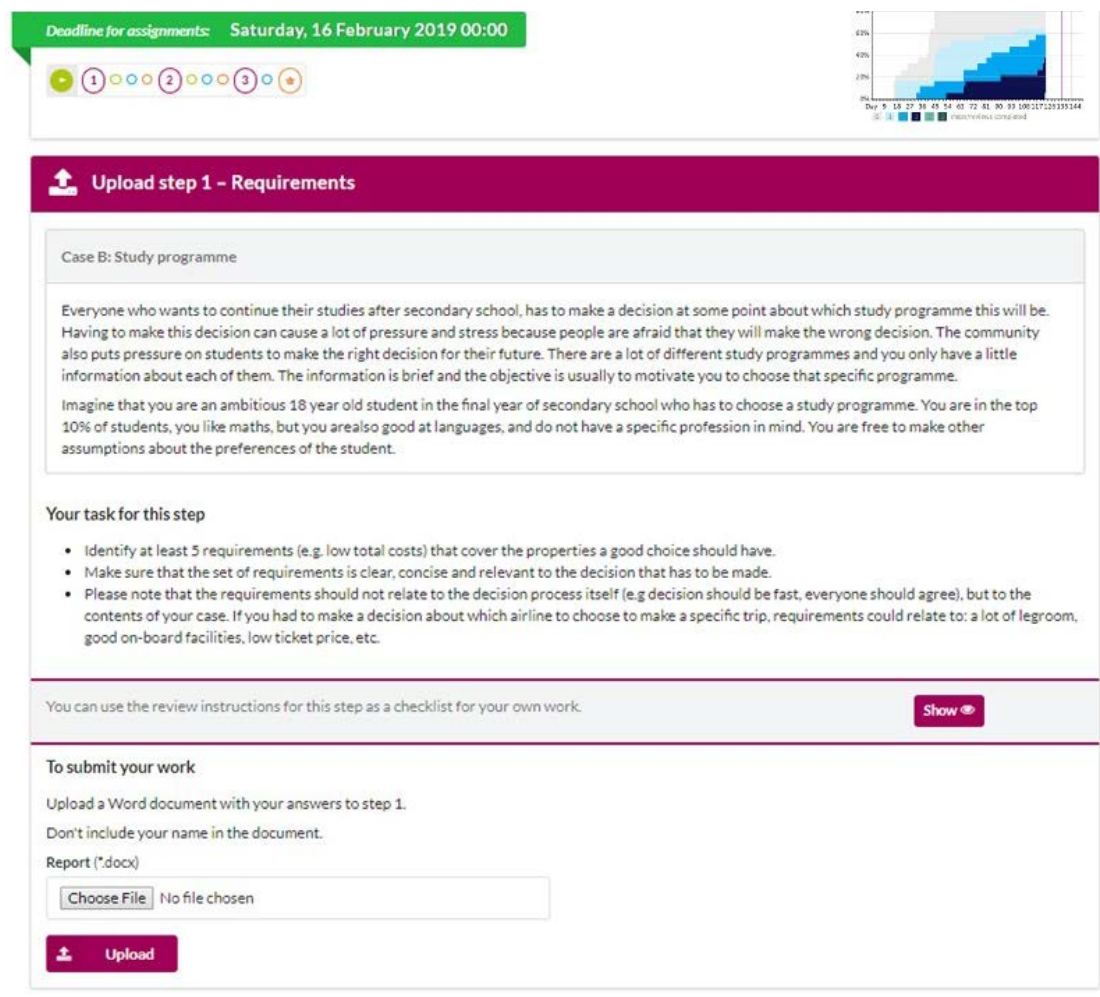

Figure 2. Screenshot of the student interface (upload task for Step 1)

Figure 2 shows what students see for an upload task: a case description, instructions on the primary task for this step, and a button to view the (itemized) review and assessment guidelines that their successor will apply. The progress bar at the top shows how far students have progressed in the relay: the large, numbered circles denote upload tasks (and hence completed steps) and final review tasks, the smaller circles denote the other tasks (start, download, and review). As the student progresses, the circles for completed tasks are filled in with their colour, and when the cursor is moved over, a balloon with the completion time pops up. The chart on the right shows the progress of all participants in the relay: time on the horizontal axis, the percentage of participants having completed a step on the vertical axis.

To improve the work and to add their own step, students edit and expand the document(s) submitted by their predecessor. These files are anonymised (stripped of metadata) each time they are downloaded. Students can enter their (itemized) reviews using a rich text editor. At the end of the review activity, students are asked to assess their predecessor's work with a star rating on a 5-point scale. Predecessors will see the review and assessment they received, and can respond, appraise the feedback on a 3-point scale $(;-)-(;)$ ), and can click the appeal button and write a rebuttal, which the instructor will then review.

\section{Developer interface}

Project relay templates define the number of steps for a relay, and for each step the specific instructions for the primary task, uploading the work, and reviewing work. Figure 3 gives an impression of how PRESTO enables developers to design templates. For each step, developers can specify the number and type of files that must be uploaded, add constraints (e.g., mandatory key words and titles to protect students against uploading the wrong document), 
and define specific review items with (optionally) partial assessments. The instructions for each of the steps should be generic so as to apply to different case sets.
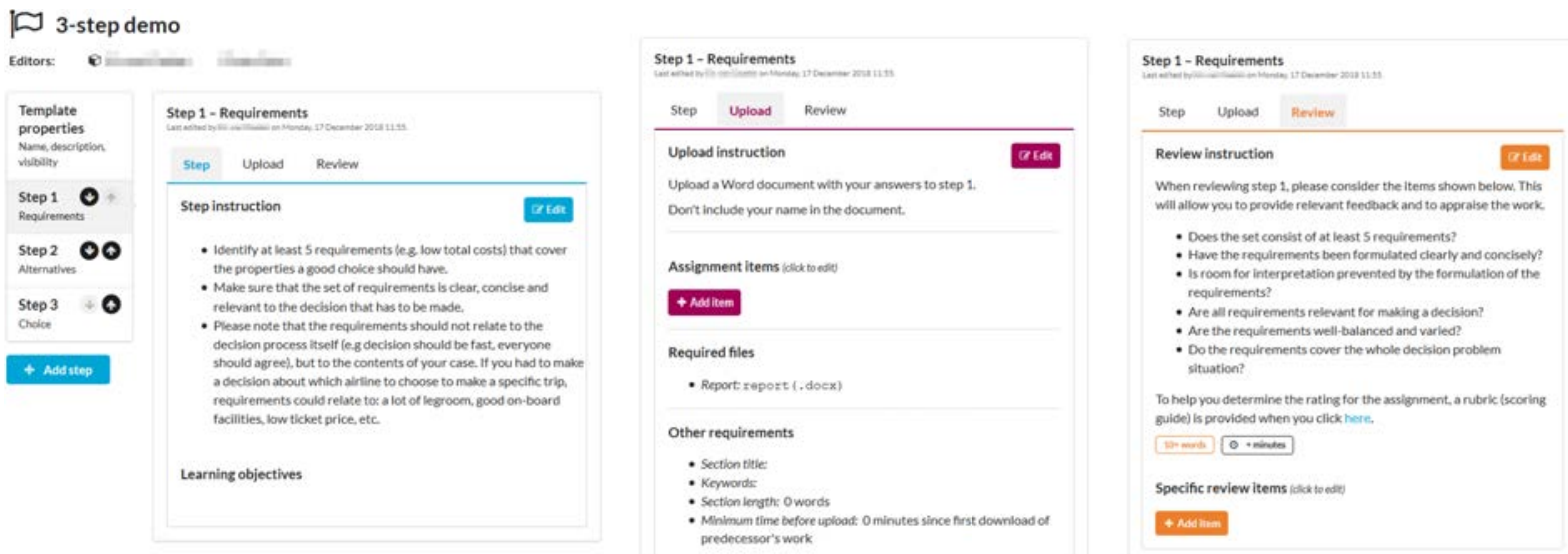

Figure 3. Partial screenshots illustrating how relay templates can be defined

\section{Instructor interface}

Figure 4 shows how PRESTO enables instructors to define case sets and create project relays.
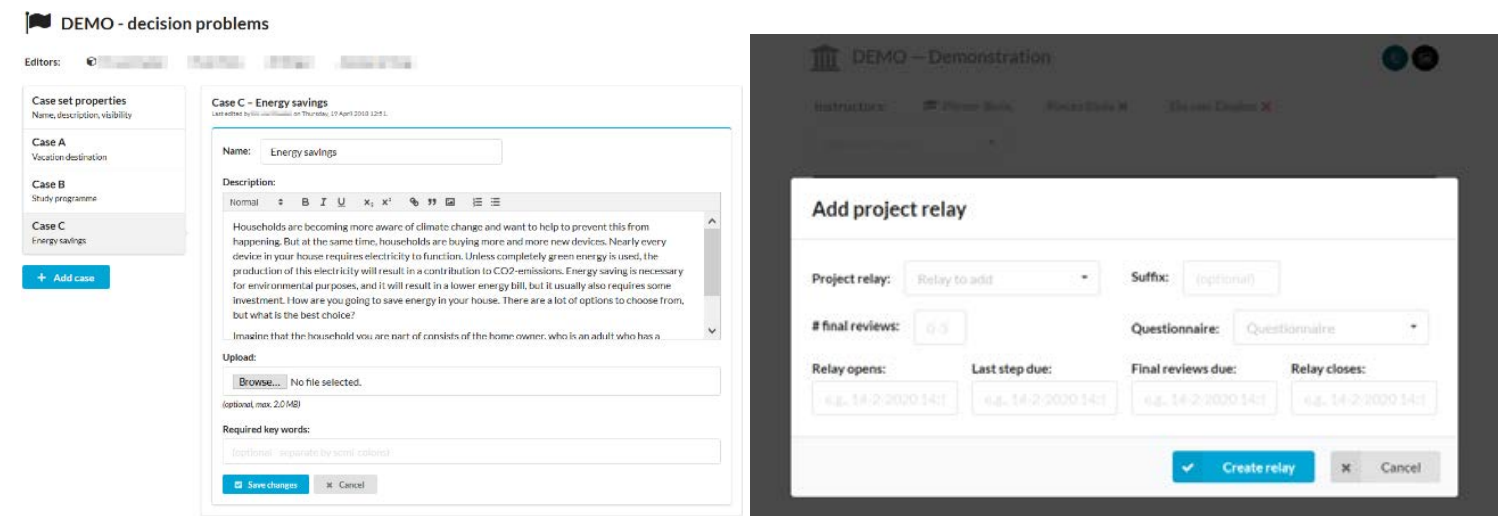

Figure 4. Partial screenshots illustrating how case sets are developed (left) and project relays created for a course (right)

\begin{tabular}{|c|c|c|c|}
\hline Name & Score & Received reviews & Given reviews \\
\hline $36 \%$ Tuesday, 11 December 2018 12:40 & 2.0 & $\begin{array}{l}\text { (C) }(A) \\
(-)\end{array}$ & (A) E \\
\hline $100 \%$ Wednesday, 19 December 2018 17:29 & 0.0 & $\begin{array}{l}\text { (c) D } F(B) \\
\text { (3) }\end{array}$ & 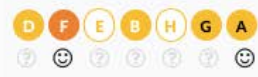 \\
\hline 95\% Wednesday, 19 December $201816: 53$ & 4.0 & 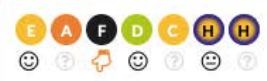 & 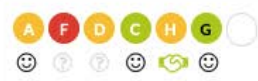 \\
\hline 95\% Monday, 17 December 2018 17:21 & 4.0 & $\begin{array}{l}\text { E (c) } F(B) \\
(-)\end{array}$ & $\begin{array}{l}\text { (C) F (D) (G) H } \\
\text { (-) \& (2) (-) }\end{array}$ \\
\hline $36 \%$ Wednesday, 12 December 2018 11:49 & -0.5 & (E) (D) & (D) 6 \\
\hline 73\% Monday, 17 December 2018 01:04 & 4.0 & (D) E E H (3) (3) & $\begin{array}{l}\text { A E } \mathrm{H} \\
\oplus \text { ही }\end{array}$ \\
\hline
\end{tabular}

Figure 5. Partial screenshot of the instructor dashboard for a project relay 
From the set starting time on, students enrolled in the course can carry out the project relay. Figure 5 gives an impression of the dashboard that allows instructors to monitor a relay. The first column in the overview shows the names of participating students, their overall progress rate, and the time of their most recent activity. The small circles indicate the completed steps, their colour reflects the similarity score computed by PRESTO's plagiarism scanner. When clicking on the name of the student, the instructor can see this student's history (uploaded work and associated interactions with predecessors, successors, and referees). The second column shows the total score of the students. When a project relay has finished, these scores can be scaled to grades once the instructors have benchmarked the work with the lowest and highest scores (cf. Figure 6). The third and fourth column show, for each completed step, the reviews received and given. The letters indicate the case worked on, while colours correspond to the rating of the step (red $=\star$, dark green $=\star \star \star \star \star$, black $=$ rejection). The icon underneath a circle reflects the reviewed student's appraisal of the feedback and rating. A hand icon indicates that the student appealed, its colour the referee's rating. Pointing hands indicate the student incurring a penalty. Moving the cursor over a circle (icon) will reveal the time of the event and the identity of the other student.

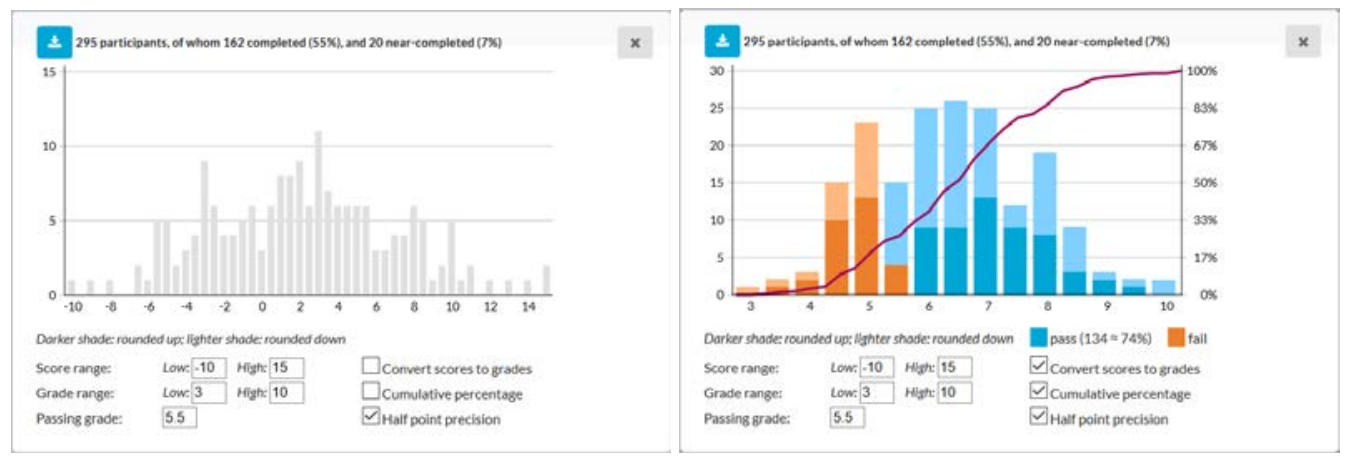

Figure 6. Scaling total scores to grades

\section{Experiences with the project relay}

We first applied the relay in the quantitative modelling course in 2013. In this course we now have run more than 15 relays with cohorts of over 200 first-year BSc students. Since 2017, the relay has also been used in a first-year MSc course on methods and tools for policy analysis with for some 40 students from 10-15 different countries. In 2018, we ran pilot relays in three different online courses (Creative Problem Solving, Next Generation Infrastructures, and Responsible Innovation) on the edX platform. This confirmed that project relays can be tailored to the subject matter and academic level of a wide range of courses, and that the PRESTO software performs well for relays with up to 350 students (the highest enrolment to date). In addition, we have also used the software in support of courses with "regular" peer review, as this corresponds with a relay comprising only a single step.

We have evaluated the project relay based on data stored in the system, such as time spent on a step, and also by means of student surveys. Students indicate that they learn a lot from the relay, and that this way of working is a good way to practice. Students also state that the workload of the relay is high. This follows in part from the effort of first improving one's 
predecessor's work before one can perform the primary task of a step. A secondary explanation of the experienced workload is that students tend to procrastinate: facing a deadline only for the final step, many students do not spread their work over the available time period (typically 2-3 weeks). This behaviour is consistent across level (BSc - MSc) and cohort $(2013-2018)$.

Students find a project relay quite stressful. Although stress is inherent to project work, and can be functional (Pope, 2015), many students resent the differential scoring mechanism makes that students can gain more points by being more critical towards their predecessor. Despite the protection offered by the opportunity to appeal, we observe much stronger emotions than those observed for absolute scoring (Vickerman, 2009). Although incentives to be critical are important for good quality work, we think we can make it less stressful by enhancing the students' capacity for evaluative judgement (Tai et al., 2017) by providing better instruction/guidelines for formulating constructive feedback (Landry et al., 2015).

\section{Conclusions and further developments}

Faced with the challenge to provide students with sufficient individual practice and feedback, while at the same time keeping the teaching burden manageable, we developed the project relay: a way of working in which students evaluate, improve, and then build on work of fellow students. The associated PRESTO software that manages the workflow of a relay will be available under an open source license.

We have used project relays in our education since 2013 in courses with large numbers of students (BSc and MSc level) with a variety of nationalities. The substantive flexibility offered by templates and case sets makes the project relay a feasible option for a broad range of courses. The open, asynchronous, double-blind relay process and the web-based, LTIcompliant PRESTO software show good potential for implementing "virtual mobility", as students enrolled in courses at different educational institutions could participate in jointly offered relays to develop generic academic skills by working in international virtual teams on case sets developed by staff from these different institutions.

The learning outcomes of the relay are quite good, but the relay can be stressful for students. We are planning to better prepare students for the review and assessment tasks with instruction and guidance, and by including formative tests at the beginning of each step to better prepare students for the substantive work. We will also explore incentives for students to write constructive reviews by making their predecessors' appreciation of the reviews (relative to the received rating) count towards the final grade in a relay. 


\section{References}

Brooks, C. M., \& Ammons, J. L. (2003). Free riding in group projects and the effects of timing, frequency, and specificity of criteria in peer assessments. Journal of Education for Business, 78(5), 268-272. doi: 10.1080/08832320309598613

Ke, F., \& Xie, K. (2009). Toward deep learning for adult students in online courses. The Internet and Higher Education, 12(3-4), 136-145. doi: 10.1016/j.iheduc.2009.08.001

Landry, A., Jacobs, S., \& Newton, G. (2015). Effective use of peer assessment in a graduate level writing assignment: a case study. International Journal of Higher Education, 4(1), 3851. doi: 10.5430/ijhe.v4n1p38

Pope, N. K. L. (2005). The impact of stress in self- and peer assessment. Assessment \& Evaluation in Higher Education, 30(1), 51-63. doi:10.1080/0260293042003243896

Tai, J., Ajjawi, R., Boud, D., Dawson, P., \& Panadero, E. (2018). Developing evaluative judgement: enabling students to make decisions about the quality of work. Higher Education, 76(3), 467-481. doi:10.1007/s10734-017-0220-3

Van Hattum-Janssen, N., \& Lourenço, J. M. (2008). Peer and self-assessment for first-year students as a tool to improve learning. Journal of Professional Issues in Engineering Education and Practice, 134(4), 346-352. doi: 10.1061/(ASCE)1052-3928(2008)134:4(346)

Vickerman, P. (2009). Student perspectives on formative peer assessment: an attempt to deepen learning? Assessment \& Evaluation in Higher Education, 34(2), 221-230. doi: $10.1080 / 02602930801955986$ 